\title{
Ultra-wideband bandpass filter using matching-lines and interdigital filter with close-coupled resonators
}

\author{
Yusuke Omote $^{\mathrm{a})}$, Takenori Yasuzumi ${ }^{\mathrm{b})}$, Tomoki Uwano ${ }^{\mathrm{c})}$, \\ and Osamu Hashimoto \\ Department of Electrical and Electronics Engineering, Aoyama Gakuin University, \\ 5-10-1 Fuchinobe, Chuo-ku, Sagamihara-shi, Kanagawa, 252-5258 Japan \\ a) omote@ee.aoyama.ac.jp \\ b)yasuzumi@ee.aoyama.ac.jp \\ c) uwano@hi-ho.ne.jp
}

\begin{abstract}
This paper presents an ultra-wideband bandpass filter (UWB BPF) using quarter-wavelength inverter-lines at both sides of a three-pole interdigital filter. The relations between the impedances of the inverter-line with the load and those of the interdigital filter connected to the inverter-line were studied. From these, the design procedures for the impedance matching at the UWB passband edges were shown. Then the designed BPF from the above results showed that the bandwidth was $24 \%$ wider than that of the sole interdigital filter by additional two conjugate matching poles. The fabricated BPF achieved the performances of the required passband by the five matching poles.
\end{abstract}

Keywords: Ultra-wideband (UWB), bandpass filter (BPF), inverterline, matching-line, conjugate matching

Classification: Microwave and millimeter wave devices, circuits, and systems

\section{References}

[1] Federal Communications Commission, "Revision of part 15 of the commission's rules regarding ultra-wideband transmission systems," First Report and Order, FCC 02.V48, April 2002.

[2] D. Kurita, K. Li, and K. Araki, "Two-layered UWB band-pass filter with transmission zeros using cross coupling," IEICE Trans. Electron., vol. J93C, no. 12, pp. 573-581, Dec. 2010.

[3] L. Li and Z. F. Li, "Side-coupled shorted microstrip line for compact quasi-elliptic wideband bandpass filter design," IEEE Microw. Wireless Compon. Lett., vol. 20, no. 6, pp. 322-324, June 2010.

[4] G. L. Matthaei, L. Young, and E. M. T. Jones, Microwave filters, impedance-matching networks, and coupling structures, Artech House, Norwood, 1980. 
[5] High frequency structure simulator (HFSS), Ansoft Corporation, Pittsburgh, PA, 2008.

\section{Introduction}

The Federal Communications Commission (FCC) authorized the unlicensed use of an ultra-wideband (UWB) wireless communication system with a frequency band from 3.1 to $10.6 \mathrm{GHz}$ in 2002 [1]. One of the key devices for the system is a bandpass filter (BPF) and many kinds of the structures were proposed such as microstrip/coplanar [2] and interdigital ones [3]. These structures realize sharp skirt characteristics by attenuation poles using a cross couplings and achieve good stopband characteristics. For improving the passband characteristics, the use of the matching section such as an inverter-line is widely known. These techniques are, however, only applicable to narrow BPFs. Thus characteristic degradations are main concern for BPFs when the passband is far and wide.

This paper presents a UWB BPF using quarter-wavelength inverter-lines for the matching to an interdigital filter. It is shown that the use of the inverter-line is a good technique for improving the filter performances in the wide passband not just around the center frequency. The impedance characteristics of the inverter-lines and those of the interdigital filter are studied at the UWB passband edges and the design procedures of the proposed UWB BPF are shown. From these procedures, the impedances are conjugatematched at the passband edges and the UWB BPF with a wide equi-ripple reflections in the passband will be designed. The comparison of the passband performances between the designed BPF and the sole interdigital filter will be shown. The fabrication of the designed BPF with required FCC indoor specs will be illustrated.

\section{Structure of the UWB BPF}

Figure 1 shows the structure of the proposed UWB BPF. The BPF con-

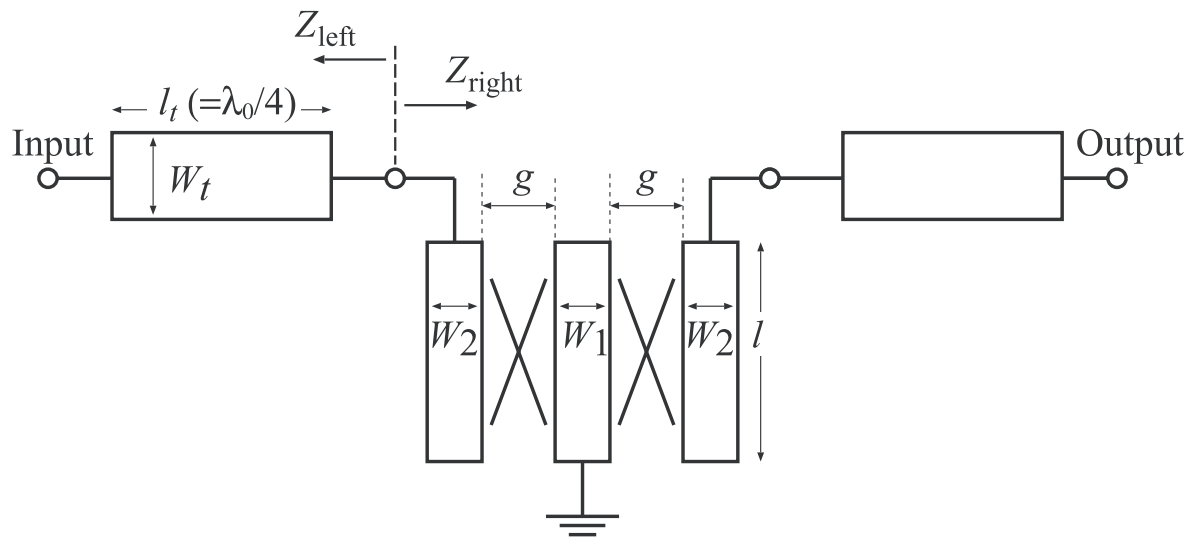

Fig. 1. Structure of the proposed UWB BPF. 
sists of an interdigital filter with close-coupled microstrip-line resonators and inverter-lines connected between the resonators and the load impedances. The resonators of the interdigital filter have widths $W_{1}$ and $W_{2}$, length $l$, and gap $g$. Each inverter-line has width $W_{t}$ and length $l_{t}$ of $\lambda_{0} / 4$, where $\lambda_{0}$ is the wavelength at the center frequency in the passband.

\section{Impedance analysis}

Impedances $Z_{\text {left }}$ and $Z_{\text {right }}$ looking into the left and right sides from the reference plane as in Fig. 1 are studied at the UWB passband edges, where the dimensions of the interdigital filter were optimized so as to have the equiripple reflections in the passband and those are set to be $W_{1}=W_{2}=0.1 \mathrm{~mm}$, $g=0.02 \mathrm{~mm}$, and $l=8.3 \mathrm{~mm}$. $l_{t}$ is $7.8 \mathrm{~mm}$ as $\lambda_{0} / 4$ at $6.85 \mathrm{GHz}$ and characteristic impedance $Z_{t}$ of the inverter-line changes from 60 to $90 \Omega$. The proposed BPF is assumed on a ROGERS RT/Duroid 5880 substrate, where the thickness of $1.57 \mathrm{~mm}$, the relative dielectric constant of $\varepsilon_{r}=2.2, \tan \theta=0.0009$, and the metal thickness of $18 \mu \mathrm{m}$. Figure 2 (a) shows frequency characteristics of $Z_{\text {left }}$ and $Z_{\text {right }}$ from 2.9 to $3.5 \mathrm{GHz}$ and from 10.5 to $11.1 \mathrm{GHz}$. From the results, $Z_{\text {left }}$ and $Z_{\text {right }}$ move monotonously by $Z_{t}$. Thus the conjugate matching can be expected at the passband edges.

Now the $Z_{\text {left }}$ and $Z_{\text {right }}$ depending on $l_{t}$ are studied where the dimensions of the interdigital filter are set as the same as the above parameters and $Z_{t}$ is $70 \Omega$. Figure $2(\mathrm{~b})$ shows the frequency characteristics of $Z_{\text {left }}$ and $Z_{\text {right }}$ when $l_{t}$ is set as $6.0,8.0$, and $10.0 \mathrm{~mm}$. From the results, $Z_{\text {left }}$ rotate clockwise because the inductance due to the inverter-line increases as $l_{t}$ is longer. $Z_{\text {right }}$ are monotonously changed by $l_{t}$ but the shifts of $Z_{\text {right }}$ are small as compared with those by $Z_{t}$.

Based on the above studies, $Z_{t}$ is decided so as to approximately conjugate $Z_{\text {left }}$ and $Z_{\text {right }}$ at the passband edges. After that $Z_{t}$ and $l_{t}$ are optimized to have the reflections below $-15 \mathrm{~dB}$ in the passband. From the results, $Z_{t}$ is given to be $75 \Omega\left(W_{t}=2.5 \mathrm{~mm}\right)$ and $l_{t}$ is set to be $8.2 \mathrm{~mm}$ changing from $7.8 \mathrm{~mm}$. Figure $2(\mathrm{c})$ shows the impedances for these resultant parameters and it is learned $Z_{\text {left }}$ and $Z_{\text {right }}$ are now matched at the point frequencies of 3.6 and $11.0 \mathrm{GHz}$. Thus the inverter-line is functioning as a wideband matching-line. Figure 3 (a) shows the characteristics of the designed BPF with the solid curves. Here the BPF has the conjugate matching poles at 3.1 and $11.0 \mathrm{GHz}$ and shows the reflection characteristics below $-15 \mathrm{~dB}$ from 2.9 to $11.2 \mathrm{GHz}$.

The designed matching-line (inverter-line) has the characteristic impedance of $75 \Omega$ and thus the interdigital filter can be assumed to be connected to $112.5 \Omega$ loads around $6.85 \mathrm{GHz}$. In Fig. 3 (a), the characteristics of the sole interdigital filter with $112.5 \Omega$ loads in the absence of the inverter-lines are also shown with the dotted curves. The sole interdigital filter has three poles in the passband and its reflection bandwidth below $-15 \mathrm{~dB}$ is from 3.6 to $10.3 \mathrm{GHz}$. With the additional two matching poles at the passband edges given by the inverter-lines, the reflection bandwidth of the proposed BPF 

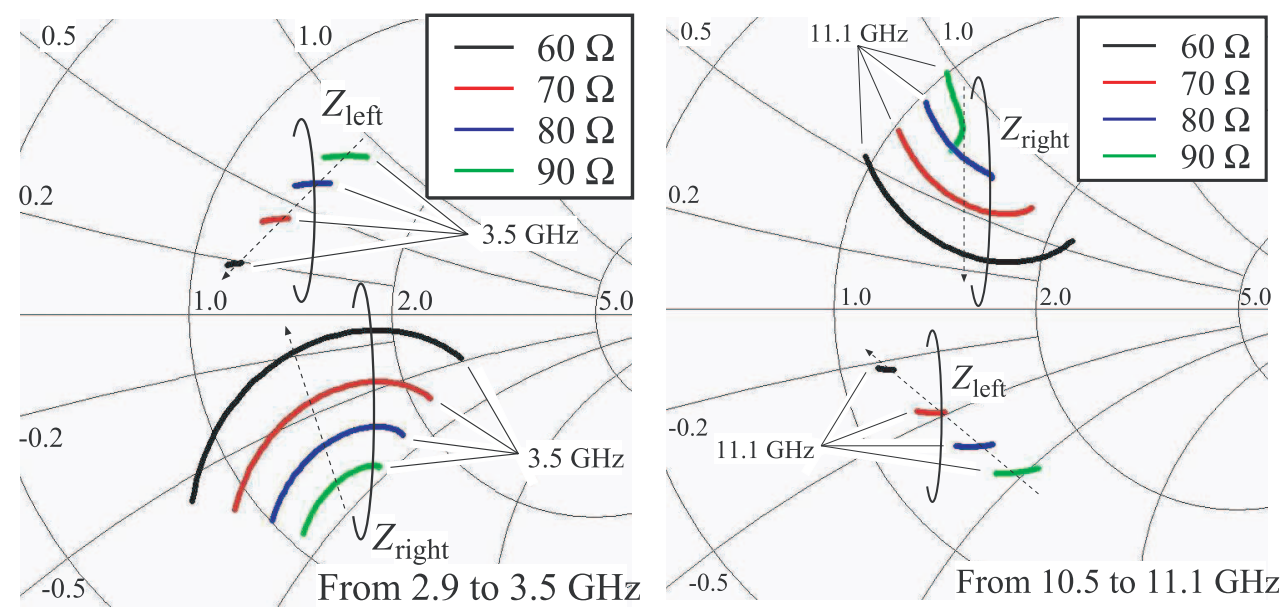

(a) Impedances by $Z_{t}$.
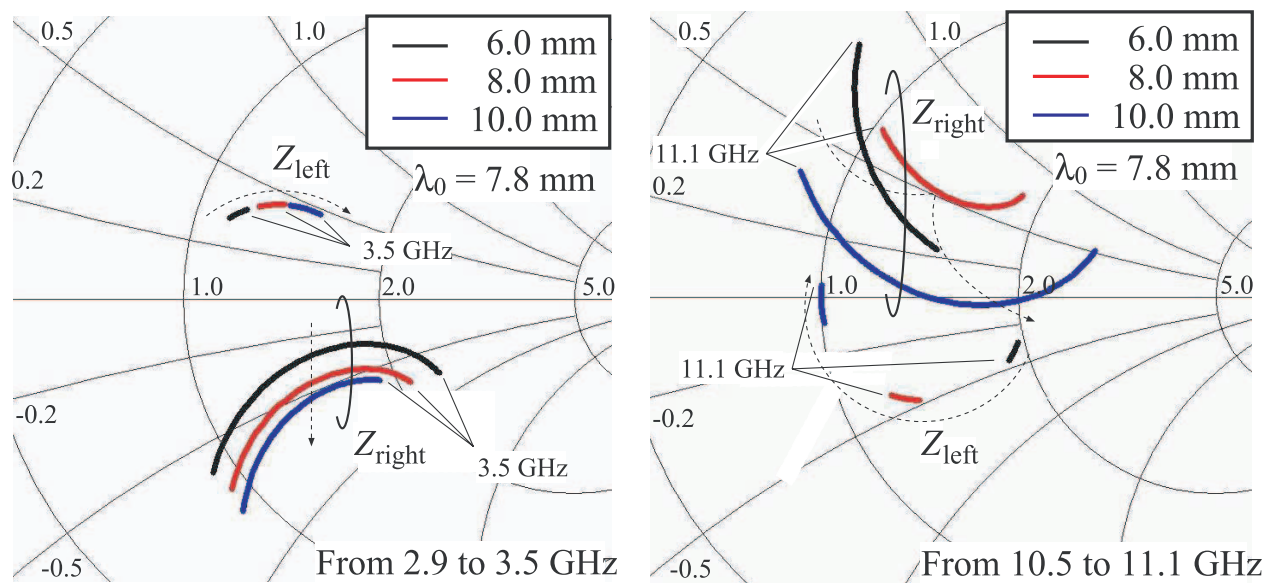

(b) Impedances by $l_{t}$.

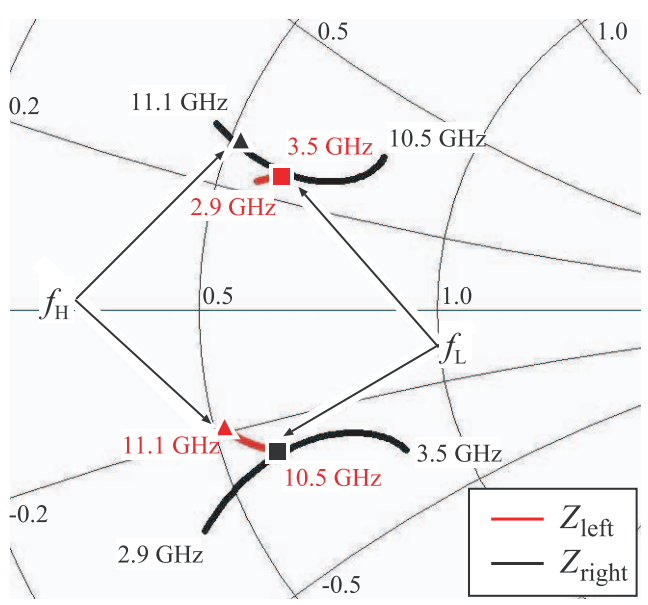

(c) $Z_{\text {left }}$ and $Z_{\text {right }}$ at the UWB passband edges.

Fig. 2. Impedance characteristics of $Z_{\text {left }}$ and $Z_{\text {right }}$.

was $24 \%$ wider than that of the sole interdigital filter.

\section{Fabrication of the UWB BPF}

(c) IEICE 2012

DOI: $10.1587 /$ elex.9.660

Received February 17, 2012

Accepted March 11, 2012

Published April 06, 2012
The BPF which satisfies the FCC indoor specs [1] was fabricated for the ex- 
periments. The dimensions of the interdigital filter were optimized to have $\left|S_{21}\right|$ of $-10 \mathrm{~dB}$ at 3.1 and $10.6 \mathrm{GHz}$ and those resulted in $W_{1}=0.16 \mathrm{~mm}$, $W_{2}=0.24 \mathrm{~mm}, g=0.1 \mathrm{~mm}$, and $l=8.0 \mathrm{~mm}$. Then the matching-lines were designed based on Fig. 2 (a) and (b) so as to make the conjugate matches for $Z_{\text {left }}$ and $Z_{\text {right }}$ in the passband edges and were given as $W_{t}=2.9 \mathrm{~mm}$ and $l_{t}=8.2 \mathrm{~mm}$. The characteristics of the UWB BPF were calculated by the electromagnetic field simulator [5]. The results are shown in Fig. 3 (b) with the solid curves where the BPF achieved $-30 \mathrm{~dB}$ equi-ripple reflection by showing the five matching poles in the passband.

The above UWB BPF was fabricated and its measured characteristics are also shown in Fig. 3 (b) with the dotted curves. From the results, it is confirmed that the BPF have the five poles in the passband. The measured characteristics have good agreements with the simulated ones and achieved

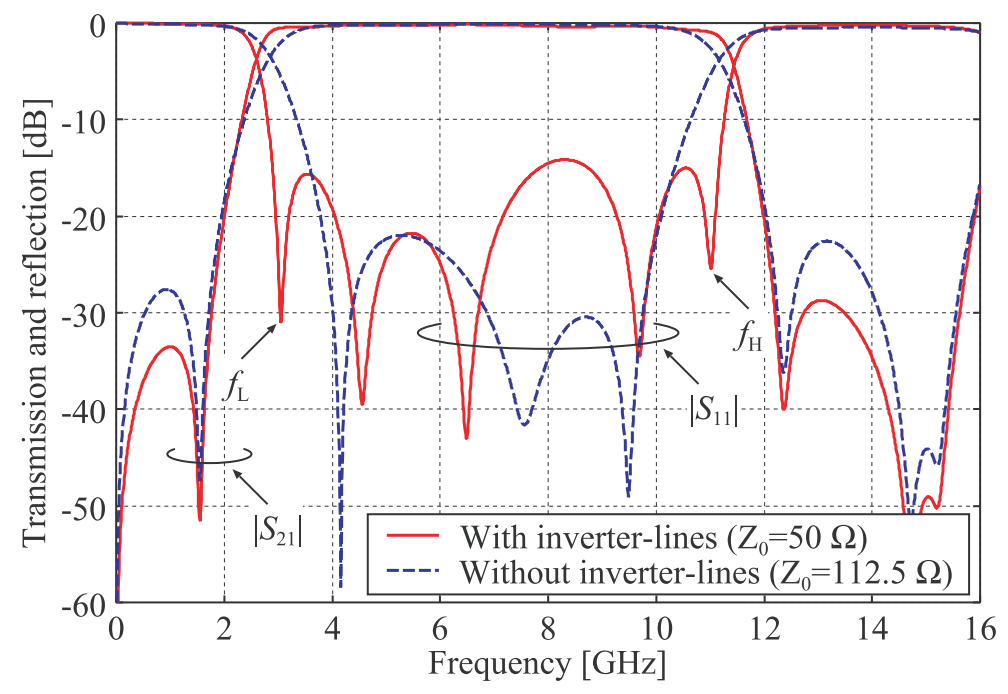

(a) Comparison for the inverter-lines.

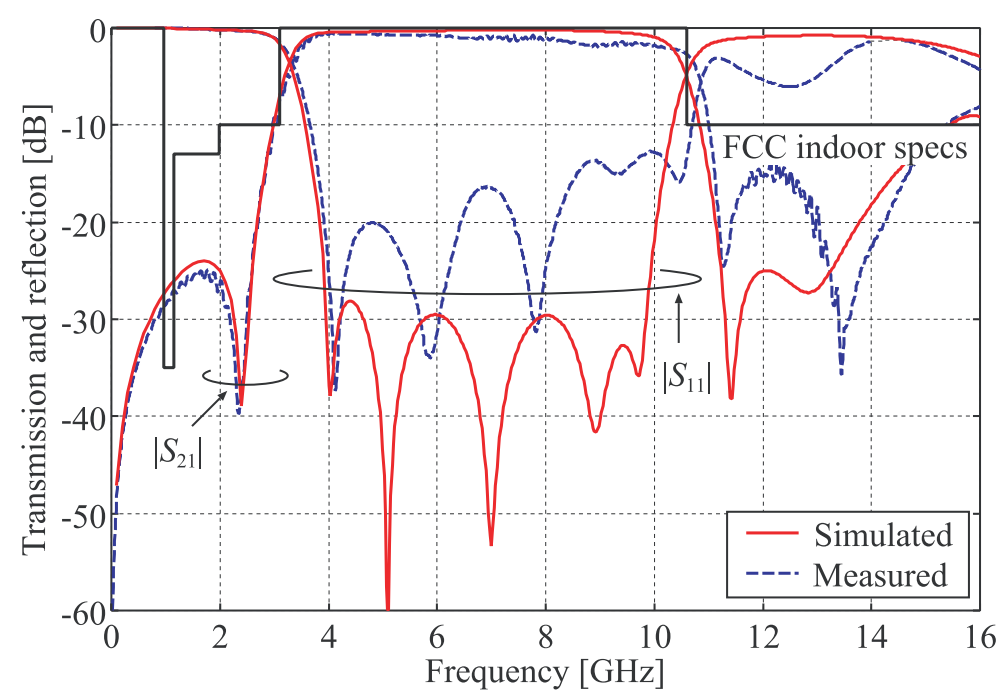

(b) Experimental results.

Fig. 3. Transmission and reflection characteristics of the proposed UWB BPF. 
the required passband bandwidth and attenuations in the higher stopband.

\section{Conclusion}

A UWB BPF using quarter-wavelength matching-lines and an interdigital filter of three finger resonators has been presented in this paper. From the impedance characteristics of the inverter-line and those of the interdigital filter connected to the inverter-line, it was shown that the inverter-line operated as the matching-line at the UWB passband edges. The reflection characteristics at the UWB passband edges were improved by using the conjugate matching poles and thus the bandwidth of the proposed BPF was $24 \%$ wider than that of the sole interdigital filter. The proposed BPF satisfying the FCC specs was designed and fabricated. The measured characteristics had the five matching poles (three and additional two) in the passband.

\section{Acknowledgments}

This work is supported by a Grant-in-Aid for The Private School High-tech Research Center Program of the Ministry of Education, Culture, Sports, Science, and Technology, Japan. 